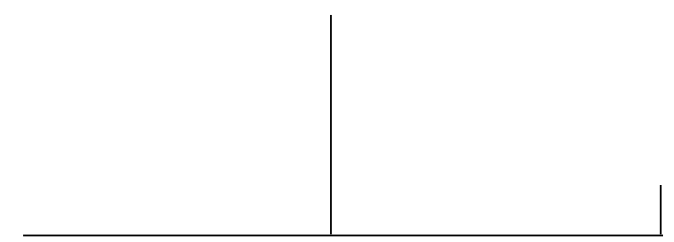

Rev. Latinoam. Psicopat. Fund., São Paulo, v. 15, n. 3, p. 549-559, setembro 2012

\title{
O conflito sexual infantil abordado de um ponto de vista psicanalítico por Egas Moniz, o pai da psicocirurgia
}

Sophie Maurissen

Mário Eduardo Costa Pereira

Em sua conferência sobre $\mathrm{O}$ conflito sexual, proferida no Congresso Luso-Espanhol das Associações para o Progresso das Ciências e publicada em 1921, Egas Moniz aplica o método psicanalítico em dois casos clínicos, utilizando para isso o divã e fazendo uso da associação livre, da associação de ideias, assim como da análise dos sonhos. Esse neurocirurgião, principalmente reconhecido por seus estudos sobre a leucotomia pré-frontal, foi a primeira pessoa a se referir e a recorrer à psicanálise em Portugal. De fato, tal como Freud, Egas Moniz analisou dois casos da literatura apoiando-se no método analítico: o caso de Júlio Dinis em 1924 e o de Camilo em 1925. No entanto, sua contribuição à psicopatologia parece assombrada por seu trabalho sobre a leucotomia, que lhe valeu o Prêmio Nobel de Fisiologia em 1949.

Palavras-chave: Egas Moniz, sexualidade infantil, perversões sexuais, psicanálise, interpretação dos sonhos 


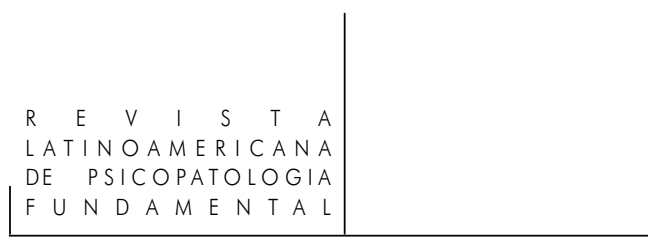

O desenvolvimento, por Egas Moniz, da angiografia cerebral (1927) constituiu, na primeira metade do século XX, um progresso decisivo no campo da imagiologia cerebral. A invenção desse método, então revolucionário, de diagnóstico de várias doenças endocranianas - como neoplasias, hemorragias, aneurismas, malformações vasculares congênitas valeu-lhe a atribuição do Prêmio de Oslo em 1945. Entretanto, apesar desse feito científico da maior relevância, Egas Moniz só se tornou mundialmente conhecido ao formalizar, em 1935, juntamente com o cirurgião Almeida Lima, a leucotomia pré-frontal no tratamento de certas doenças mentais, notadamente a depressão grave, o transtorno obsessivo-compulsivo e, mesmo, a esquizofrenia. Essa nova forma de intervenção cirúrgica visando modificar a manifestação de fenômenos psicopatológicos, pela qual the foi atribuído o Prêmio Nobel de Fisiologia em 1949, fez desse médico o pai da psicocirurgia e permitiu-lhe a entrada nas Academias de Ciências de Lisboa, Rio de Janeiro, Paris, Madrid, Estrasburgo, Buenos Aires, Nova Iorque e Lyon. Ainda que tenha sido recebida com muitas reservas, essa técnica foi largamente utilizada em todo o mundo nas décadas seguintes, em particular nos Estados Unidos, chegando ao Brasil já em 1936 por intermédio do neurologista Aloysio Mattos Pimenta. Além de suas finalidades terapêuticas específicas, esse procedimento neurocirúrgico abriu um novo caminho para o estudo da fisiologia no sistema nervoso central (Masiero, 2003, p. 549). Dessa forma, muitas foram as honrarias e grande o reconhecimento científico atribuídos ainda em vida a Egas Moniz por sua descoberta. Contudo, após uma forte controvérsia sobre seus riscos e sobre suas implicações éticas, a psicocirurgia deixou de ser praticada nos anos 1960, retornando com novo vigor nos tempos atuais, sobretudo como tratamento extremo para o transtorno obsessivo-compulsivo ou para certas formas de transtornos alimentares.

Egas Moniz realiza seus estudos médicos na Faculdade de Medicina da Universidade de Coimbra. A partir de 1902, aprofunda a sua formação na área da Neurologia na França, mais especificamente em Bordeaux, com Pitres e Régis, e em Paris com Pierre Marie, Dejerine e Joseph Babinski. Aos 28 anos, recém-doutorado e especialmente dedicado à Neurologia, passa a lecionar, durante nove anos, Anatomia e Fisiologia na Faculdade de 
Coimbra. Em seguida, é transferido para a recém-criada Faculdade de Medicina da Universidade de Lisboa onde ocupa a cátedra de Neurologia. Pouco depois, abandona a medicina por uma carreira política e somente nove anos mais tarde retorna à vida médica e universitária em Lisboa, onde ficou de 1921 a 1944.

Apesar de sua orientação científica e técnica claramente neurobiológica, é de realçar que Egas Moniz escolheu como tema de tese de doutoramento a sexualidade, sujeito "tabu" que demonstra o seu "gosto pelo risco e por estar à frente dos outros" (cf. Pereirinha, 2006, p. 78). De fato, com apenas 27 anos e em fase de provas de doutoramento, atreveu-se a abordar um tema incômodo para a universidade, estudando, contra a opinião dos seus mestres, questões polêmicas como a homossexualidade, a prostituição etc. Sua tese, publicada sob a forma de um livro, tornou-se um verdadeiro êxito editorial, pois dele existem 19 edições que foram atualizadas à luz de novos conhecimentos, entre outros o da psicanálise. Reputado enquanto sexólogo, Egas Moniz mostrou desde 1922 um vivo interesse pelo estudo da sexualidade, em suas diferentes dimensões, e da hipnose. Daí, a sua atração pela psicanálise parecer "lógica" (cf. Luzes, 1997, p. 11).

Seus trabalhos científicos referentes à angiografia cerebral e à leucotomia pré-frontal, pela abundância e novidade da informação, constituem os que mais interesse têm despertado nos estudiosos de sua obra. As diversas críticas apontadas a esses estudos parecem assombrar a obra revolucionária desse neurocirurgião no campo da psicopatologia. Entretanto, é interessante constatar que desde 1935 ele salientava os efeitos secundários desse método de intervenção e sustentava a posição segundo a qual esse tratamento radical só poderia ser aplicado quando todos os outros métodos terapêuticos tivessem sido comprovados ineficazes.

Depois dos êxitos dos diagnósticos da angiografia e dos progressos consequentes da Neurologia, Egas Moniz, curioso pelo desconhecido e ávido por desbravar novos domínios, interessa-se então por um território mais vasto: o estudo das doenças mentais. O Centro de Estudos Egas Moniz, do qual será presidente, fundado em 1950 no Hospital psiquiátrico Júlio de Matos, demonstra desde logo o seu interesse pelo campo da psicopatologia.

De um ponto de vista psicopatológico, Egas Moniz foi o primeiro autor português a considerar a perversão sexual em crianças, demonstrando manifestações psicogenitais poderosas e precoces, nas quais prevalece um desequilíbrio das pulsões parciais relacionadas com as zonas erógenas. As perversões sexuais são assim, pela primeira vez em Portugal, tomadas em conta como "verdadeiras psicopatias" (cf. Egas Moniz, 1922, p. 101).

No que diz respeito aos diversos sintomas somáticos e psíquicos ligados à função sexual, ele defende uma correlação entre o funcionamento das glândulas genitais e da glândula tireoide. Ousa então a interpretação seguinte: "a luta entre 
a necessidade da satisfação da libido" (consequência das glândulas endócrinas e de outros fatores orgânicos) e as "oposições de toda a ordem vindas do meio exterior", dão lugar a "muitas perturbações mentais nas neuroses e até nas psicoses” (cf. Egas Moniz, 1921, p. 73). O autor introduz, assim, uma perspectiva inédita ao estudo da sexualidade na literatura portuguesa, dando ênfase ao "estudo psicológico das tendências infantis no sentido da sexualidade" desenvolvido por Freud. Egas Moniz considera que o instinto sexual aparece na idade infantil (formadora da libido), dado que as glândulas ligadas a esse instinto estão presentes desde o nascimento do ser humano e não apenas na puberdade, como se pensava ainda nessa época e como ele próprio tinha previamente afirmado em 1901, na Fisiologia da vida sexual.

Em seu tex to intitulado A vida sexual, cujo Prólogo confronta o neurologista português com a psicanálise de Freud, Egas Moniz revela um espírito científico ímpar alicerçado na assimilação atenta e crítica das grandes lições dos pioneiros da sexologia, tais como Krafft-Ebing, Moll e Freud. Nesse texto o autor visa atrair a atenção dos psicólogos e dos neuropatologistas para a teoria freudiana, partilhando os resultados da investigação psicanalítica que adquiriu diretamente pela leitura aprofundada da obra de Freud. A teoria da sexualidade exposta por Egas Moniz nessa obra, particularmente na seção da Patologia da vida sexual, analisa a homossexualidade masculina e feminina como psicopatologias do comportamento sexual, a partir de casos clínicos nos quais observa graves efeitos na saúde e no bem-estar dos pacientes. Esse médico possuía um material clínico suficiente para propor uma tipologia diferencial da homossexualidade masculina, nomeadamente, a dicotomia masculino-feminino, as atividades e preferências sexuais, as ocupações profissionais atípicas, a sexualidade infantil etc. No entanto, apesar de ter vislumbrado a problemática das diferenças sexuais e de gênero, Egas Moniz foi excessivamente "redutor" por sua generalização a partir dos seus casos clínicos, cujas características históricas e culturais e seu estatuto contingente não foram suficientemente levadas em conta.

No campo da psicanálise, o caso desse neurocirurgião é duplamente interessante, pois o primeiro trabalho científico nessa área em Portugal é de sua autoria e foi ele quem primeiro falou de Freud nesse país, no seu texto intitulado "As bases da psicanálise" (1915). De fato, os primeiros psicanalistas portugueses demonstram uma importante consideração a esse notável pesquisador atribuindo-lhe o lugar histórico de primazia na introdução da psicanálise em Portugal. Egas Moniz, ao partilhar o seu interesse pelo método de investigação psicanalítica nas suas aulas de neurologia, demonstra o seu profundo conhecimento dos trabalhos de Freud e a sua vontade de tomar em consideração as técnicas analíticas tanto no estudo das doenças mentais como na formação médica. Nos seus textos intitulados "As bases da psicanálise" (1915), "O conflito sexual” (1921), "A vida se- 


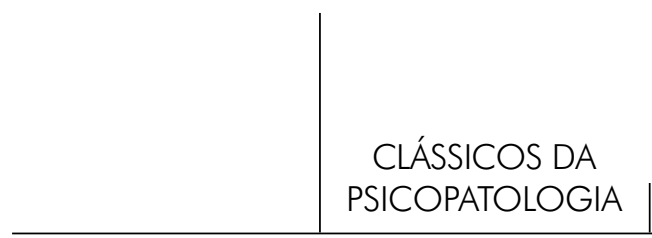

xual" (1922) e "Júlio Dinis e a psicanálise" (1924), Egas Moniz apresenta em Portugal a primeira abordagem sistemática sobre os conceitos fundamentais do método analítico freudiano. Nessas obras reside uma tentativa de divulgar as ideias de Freud no meio científico e cultural português. O texto de Egas Moniz em que esta tentativa se apresenta de forma mais clara é o de sua lição inaugural do curso de Neurologia (disciplina pela primeira vez obrigatória na licenciatura de Medicina em Portugal) proferida em três partes: a primeira destinada ao estudo da psicanálise; a segunda à exposição dos métodos de investigação psicanalítica; e a terceira às suas aplicações clínicas.

Egas Moniz foi autor de uma extensa série de publicações de excelente nível, "sem dúvida as melhores dadas à estampa na primeira metade do século XX em Portugal" (cf. Luzes, 1997, p. 11), de que selecionamos um texto proferido no Congresso Luso-Espanhol das Associações para o Progresso das Ciências e publicado em 1921. Essa obra é, a diversos títulos, brilhante, erudita e muito avançada para o seu tempo em Portugal, tal como pretendemos demonstrar.

Nesse artigo, Egas Moniz propõe distinguir as "duas grandes forças que movimentam e impulsionam a humanidade" e que constituem a base da atividade física e psíquica. São elas o "instinto da nutrição e da conservação pessoal" e o "instinto sexual ou da reprodução" (cf. Egas Moniz, 1921, p. 67). No início de sua conferência, o orador sublinha o papel central do instinto sexual na atividade mental do ser humano considerando-o como fonte primária de toda a energia psíquica. Tal como Freud, esse neurocirurgião refere-se à sexualidade infantil defendendo que a libido na criança pequena é obtida por meio da satisfação pela excitação das zonas erógenas (mucosa anal, labial, algumas regiões da pele etc.). A exemplificação da difícil distinção nos primeiros momentos de existência do ser humano entre o instinto da nutrição (mamar) e sexual (prazer erótico de sucção), a que Egas Moniz se refere no início do seu texto, surge então pela referência ao prazer da nutrição e à satisfação erótica que a criança ressente no ato de sucção, tal como Freud demonstrou em 1905. Assim, o autor dá ênfase ao valor atribuído por Freud ao problema sexual, apoiando-se no caso clínico de Anna O. apresentado por Breuer, que inicialmente utilizara, sem sucesso, a hipnose com essa paciente, mas que termina por alcançar uma clara melhora de seus sintomas com o emprego daquele método que paciente e terapeuta foram criando espontaneamente e que nomearam talking cure.

Egas Moniz apresenta em seguida o caso clínico de uma paciente de trinta anos que sofria de paralisia dos membros inferiores. $\mathrm{O}$ autor indica que, face à ineficácia dos outros meios - entre os quais os tratamentos psicoterápicos tradicionais -, ele termina por recorrer à psicanálise, dedicando-se a um trabalho interpretativo relativo não só aos "dizeres" da paciente, mas também a todos os detalhes que puderam surgir nas consultas. Através desse caso clínico, que "pa- 
tenteou as vantagens" do processo analítico, ele partilha os "grandes triunfos clínicos" devidos à psicanálise dado que, na sua opinião, o conflito sexual "assunto ainda pouco divulgado nos países latinos" na sua época, está diretamente ligado às perturbações da vida sexual (1921, p. 69-70). Diz ainda que por não ser tomado em consideração esse método de investigação analítico, que permite "investigar as tendências afetivas, especialmente nas psiconeuroses", diversas neuroses se encontram sem cura ou ainda que surgem complicações de certas situações clínicas por estas não terem sido atempada e devidamente interpretadas (p. 70). $\mathrm{Na}$ opinião do autor, todos sentem (incluindo os médicos), uma "repulsa em se ocuparem de questões que se tornam penosas quando são aprofundadas" (p. 70). Estabelece então um paralelo entre o ataque à sua tese de doutoramento sobre a Vida sexual e esse sentimento de "repugnância natural" que deve ser vencido (p. 70). Segundo ele, suas doutrinas feriam mais pelo fato de serem trazidas a público do que propriamente pelo seu conteúdo. No entanto, muitas das críticas à obra de Egas Moniz referiam-se à sua concepção neomaltusianista face a sexualidade.

No texto que propomos estudar no âmbito deste artigo, Egas Moniz apresenta o "conflito" que anuncia no título da sua conferência, em que se encontram por um lado o desejo da satisfação sexual e, por outro, "os fatores de ordem elevada que tendem a reprimi-lo" tal como as influências educativas inibitórias da sexualidade infantil (vergonha, pressões morais e religiosas). Em sua apresentação parece estabelecer um paralelo entre estas últimas e o conceito freudiano de Censura que visa recalcar o reprimido e as tendências sexuais mórbidas no inconsciente. Sustenta que este conflito não tem apenas lugar na vida infantil, mas também adulta na qual "o receio das consequências que podem resultar da livre expansão do impulso libidinoso" se acrescenta aos outros obstáculos de ordem elevada (p. 78).

Para o estudo aprofundado do "conflito sexual", Egas Moniz retoma os conceitos freudianos de inconsciente, consciente e pré-consciente, os quais já havia exposto na sua "Lição do curso de neurologia" (1915). Apoiando-se na primeira tópica de Freud, teorizada em 1900, ele aponta algumas críticas a essa teoria. Em sua opinião, a origem das neuroses não reside exclusivamente nos traumas sexuais da infância, tendo assim a concepção freudiana de ser contextualizada. Considera também que a escola de Freud apresenta conclusões exageradas quando postula que "psiconeuroses e perversões sexuais são uma e a mesma doença resultante da perturbação do desenvolvimento psicossexual", pois constata que, por vezes, estas duas não coincidem, mas se associam (cf. Egas Moniz, 1915, p. 60). Afirma ainda que a interpretação dos sonhos dos neuropatas "é uma das mais interessantes aquisições da escola de Freud" apesar de existirem "alguns exageros na sua complexa tessitura interpretativa" (cf. Egas Moniz, 1921, p. 80). No en- 
tanto, é importante realçar que a concepção de Egas Moniz relativa à atividade onírica difere em parte da de Freud. Com efeito, o primeiro sustenta que "o sonho é uma linguagem que exprime as ideias que contém através de imagens concretas" enquanto o segundo afirma que a essência do sonho é a realização de um desejo infantil reprimido.

É de realçar um elemento muito interessante na apresentação de Egas Moniz que, sem se referir explicitamente ao termo de complexo de Édipo, inicialmente teorizado por Freud na sua carta a Fliess de 15 de outubro de 1897, descreve no menino e na menina "o fato que a criança, em geral, se sente mais atraída pelo progenitor do sexo oposto" (p. 74). Aborda também as "manifestações sadistas", mais frequentemente presentes nas crianças do sexo masculino e as "manifestações masoquistas", mais comuns nas do sexo feminino, tal como Freud o sustentou em 1905. Egas Moniz avança igualmente, a ideia de que "a mulher mais equilibrada apresenta tendências masoquistas exteriorizadas principalmente na natural submissão ao homem, produto da educação que avigora uma predisposição instintiva" (p. 74). Nesta sua afirmação, está presente a hipótese de uma disposição natural da mulher ao masoquismo, tal como Freud afirmará três anos depois quando teoriza o masoquismo feminino a potiori (cf. Freud, 1924, p. 13).

Na sua conferência sobre "O conflito sexual", Egas Moniz parece trazer mais do que uma resposta ao tema anunciado no título da sua apresentação. De fato, o autor aborda esse conceito de um ponto de vista inesperado para a literatura da sua época, não só no campo da neurologia, mas também no da psicologia, demonstrando assim o seu grande interesse e capacidade em estudar diversos campos de investigação desenvolvidos em todo o mundo. Esse grande neurocirurgião não só aplica e estuda de forma aprofundada essa nova área da psicanálise, como também constata os limites desta última sublinhando que "muitos neuropatas não se curam completamente" por esse método. Realçando a importância e a vantagem da divulgação da psicanálise, Egas Moniz considera que a ignorância dessa disciplina representa uma "lacuna" nos programas de ensino médico português da época e tenta contribuir para essa formação ao partilhar e difundir o método analítico.

Agradecimentos: Desejamos agradecer especialmente ao Professor Doutor João Lobo Antunes a sua amável colaboração e disponibilidade.

\section{Referências}

Berrios, G.E. The origins of psychosurgery. History of Psychiatry, n. 8, p. 61-82, 1997.

Egas Moniz (1915). Lição do curso de neurologia. As bases da psicanálise. In: Cem 
anos de psicanálise. Lisboa: ISPA, 1997. p. 51-64.

Egas Moniz (1921). O conflito sexual. In: Cem anos de psicanálise. Lisboa: ISPA, 1997. p. 65-85.

Egas Moniz (1922). A vida sexual. In: Cem anos de psicanálise. Lisboa: ISPA, 1997. p. 87-104.

Egas Moniz (1924). Júlio Dinis e a psicanálise. In: Cem anos de psicanálise. Lisboa: ISPA, 1997. p. 105-116.

Freud, S (1924). Le problème économique du masochisme. In: Euvres Complètes de Freud. Paris: Presses Universitaires de France, 2006. v. 17, p. 11-23.

Lobo Antunes, J. Egas Moniz. Uma biografia. Lisboa: Gradiva, 2010.

LuZes, P. Estudo introdutivo. In: Cem anos de psicanálise. Lisboa: ISPA, 1997. p. 1-17.

Masiero, A. L. A lobotomia e a leucotomia nos manicômios brasileiros. História

Ciências Saúde-Manguinhos, Rio de Janeiro, v. 10, n. 2, p. 549- 572, 2003.

Pereirinha, F. Recenções. Afreudite, Lisboa , v. 2, n. 3/4, p. 77-96, 2006.

\section{Resumos}

(Infantile Sexual Conflict Approached from a Psychoanalytic Point of View by Egas Moniz, the Father of Psychosurgery)

During his conference on sexual conflict, given at the in Luso-Spanish Congress of Associations for Progress of Science, and published in 1921, Egas Moniz explained his use of the analytic method and device (the couch) to present the study of two clinical cases. He also discussed the principle of free association, association of ideas and the interpretation of dreams. Moniz was a neurosurgeon known primarily for his studies on prefrontal leucotomy, and was the first author to refer to and use psychoanalysis in Portugal. Like Freud, Moniz analyzed two cases in the literature in support of the analytical method: the case of Júlio Dinis in 1924 and that of Camilo in 1925. Nevertheless, his work on leucotomy, which earned him the Nobel Prize in Physiology in 1949, appears to have cast a shadow on his contributions to psychopathology.

Key words: Egas Moniz, infantile sexuality, sexual perversions, psychoanalysis, dream interpretation

(Le conflit sexuel infantile étudié d'un point de vue psychanalytique par Egas Moniz, le père de la psychochirurgie)

Dans sa conférence "Le conflit sexuel", tenue au Congrès Luso-Espagnol des Associations pour le Progrès des Sciences et publiée en 1921, Egas Moniz applique la mé- 
thode psychanalytique à deux cas cliniques en utilisant le dispositif du divan, le principe des associations libres, l'association d'idées ainsi que l'analyse des rêves. Ce neurochirurgien, reconnu en premier lieu pour ses études sur la leucotomie préfrontale, fut le premier auteur à faire référence et à recourir à la psychanalyse au Portugal. En effet, tout comme Freud, Egas Moniz analysa deux cas de la littérature à l'aide de la méthode analytique: le cas de Júlio Dinis en 1924 et celui de Camilo en 1925. Néanmoins, ses travaux sur la leucotomie, qui lui ont valu l'attribution du prix Nobel de physiologie en 1949, semblent avoir offusqué sa contribution à la psychopathologie.

Mots clés: Egas Moniz, sexualité infantile, perversions sexuelles, psychanalyse, interprétation des rêves

(El conflicto sexual infantil desde el punto de vista psicoanalítico de Egas Moniz, padre de la psicocirurjía)

En una conferencia sobre el conflicto sexual proferida en el Congreso Luso-español de las Asociaciones para el Progreso de las Ciencias y publicada en 1921, Egas Moniz aplica el método psicoanalítico en dos casos clínicos, utilizando para eso el diván, haciendo uso de la asociación libre de ideas, así como del análisis de sueños. Este neurocirurjano, reconocido principalmente por sus estudios sobre la leucotomia prefrontal, fue la primera persona a referirse y recurrir al psicoanálisis en Portugal. De hecho, tal como Freíd, Egas Moniz analisó dos casos de la literatura apoyándose en el método psicoanalítico: el caso de Julio Dinis en 1924 y el de Camilo en 1925. Sin embargo, su contribución a la sicopatología parece ensombrecida por su trabajo sobre la leucotomia, lo que le valió el Premio Nobel de Fisiología en 1949.

Palabras clave: Egas Moniz, sexualidad infantil, perversiones sexuales, psicoanálisis, interpretación de sueños

(Betrachtung des sexuellen Konfliktes in der Kindheit, aus der Perspektive der Psychoanalyse, von Egas Moniz, Vater der Psychochirurgie)

In seinem Vortrag "Der sexuelle Konflikt" auf dem portugiesisch-spanischem Kongress der Verbände zum Fortschritt der Wissenschaften, der 1921 veröffentlicht wurde, wendet Egas Moniz die psychoanalytische Methode in zwei klinischen Fällen an. Dazu verwendet er den Diwan und bedient sich der freien Assozation, der Ideenassozation, sowie der Traumdeutung. Dieser Neurochirurge, besonders anerkannt wegen seiner Studien bezüglich der präfrontalen Leukotomie, war der erste Mensch, der sich auf die Psychoanalyse bezog und sie in Portugal anwendete. Ähnlich wie Freud, hat auch Egas Moniz zwei Fälle der Literatur mit Hilfe der analytischen Methode untersucht: der Fall von Júlio Dinis, 1924 und von Camilo, 1925. Doch sein Beitrag zur Psychopathologie, scheint von seiner Untersuchung bezüglich der Leukotomie beeinflusst zu sein, wofür er 1949 den Nobelpreis für Physiologie erhielt.

Schlüsselwörter: Egas Moniz, Sexualität in der Kindheit, sexuelle Perversionen,

Psychoanalyse, Traumdeutung

Rev. Latinoam. Psicopat. Fund., São Paulo, v. 15, n. 3, p. 549-558, setembro 2012 
Citação/Citation: Maurissen, S.; Pereira, M.E.C. O conflito sexual infantil abordado de um ponto de vista psicanalítico por Egas Moniz, o pai da psicocirurgia.. Revista Latinoamericana de Psicopatologia Fundamental, São Paulo, v. 15, n. 3, p. 549-558, setembro 2012.

Editor do artigo/Editor: Prof. Dr. Mario Eduardo Costa Pereira

Recebido/Received: 20.7.2012 / 7.20.2012 Aceito/Accepted: 25.7.2012 / 7.25.2012

Copyright: () 2009 Associação Universitária de Pesquisa em Psicopatologia Fundamental/ University Association for Research in Fundamental Psychopathology. Este é um artigo de livre acesso, que permite uso irrestrito, distribuição e reprodução em qualquer meio, desde que o autor e a fonte sejam citados/This is an open-access article, which permits unrestricted use, distribution, and reproduction in any medium, provided the original author and source are credited.

Financiamento/Funding: Os autores declaram não ter sido financiados ou apoiados/The authors have no support or funding to report.

Conflito de interesses/Conflict of interest: Os autores declaram que não há conflito de interesses/The authors declare that has no conflict of interest.

\section{Sophie Maurissen}

Aluna do Master em psicologia clínica do Laboratoire de Psychopathologie Clinique: Langage et subjectivité da Aix-Marseille Université (França)

Les Chènevriers

F-05300 Antonaves, France

e-mail:smaurissen@gmail.com

\section{Mario Eduardo Costa Pereira}

Psiquiatra; psicanalista; Diretor científico e professor titular de Psicopatologia Clínica do Laboratoire de Psychopathologie Clinique: Langage et Subjectivité da Aix-Marseille Université (Marseille, França); Doutor em Psicopatologia Fundamental e Psicanálise pela Universidade de Paris 7 (Paris, França); Membro da Associação Universitária de Pesquisa em Psicopatologia Fundamental (São Paulo, SP, Brasil); Autor dos livros Pânico e desamparo (São Paulo: Escuta, 1999) e Psicopatologia dos ataques de pânico (São Paulo: Escuta, 2003); Membro do departamento de Psicanálise do Instituto Sedes Sapientiae (São Paulo, SP, Brasil).

Laboratoire de Psychanalyse et Psychopathologie Clinique Université de Provence - Centre Saint-Charles

Case 37

3, place Victor Hugo

13331 Marseille cedex 3 France

e-mail: mario-eduardo.costa-pereira@ univ-provence.fr 